\title{
Reflection of ultrasonic waves at a liquid-cubic-solid interface
}

\author{
Abdullah Atalar \\ Middle East Technical University, Electrical Engineering Department, Ankara, Turkey
}

(Received 16 May 1982; accepted for publication 27 September 1982)

The results of numerical calculations are presented for the reflection coefficient of sound waves incident on a liquid-cubic-solid interface. The reflection coefficient is calculated numerically for the (001) face of various cubic crystals. It is found that for certain orientations there is a null in the reflection coefficient. At this orientation all the power is coupled into a quasishear wave inside the solid. An explanation based on impedance theory is presented. The explanation given predicts that there might be reflection nulls for other liquid-solid interfaces where the solid is any anisotropic solid not just cubic.

PACS numbers: 43.20.Fn, 68.45. - v, 43.20.Bi

\section{INTRODUCTION}

The reflection coefficient at a liquid-solid interface proved to be an important quantity for the characterization of the solid. It is found that the material properties influence the reflectance of bounded beams incident on the interface near the Rayleigh critical angle. ${ }^{1-3}$ The transition of the reflection coefficient phase causes a lateral displacement of the incident beam known as the "Schoch displacement." In fact this displacement is proportional to the slope of the phase transition. ${ }^{4}$

Most of the work relating to the reflection coefficient at a liquid-solid interface was limited to isotropic solids. For that case explicit expressions can be derived for the reflection coefficient and also for the Schoch displacement. For anisotropic media an analytic expression is hard to obtain and therefore numerical methods must be utilized. In this paper the simplest anisotropic crystal will be considered: the reflection coefficient will be calculated for the (001) face of a cubic crystal. The media will be assumed lossless and piezoelectricity will be neglected.

\section{METHOD OF SOLUTION}

Consider the geometry of Fig. 1 where the axes are chosen such that the $x$ axis coincides with [100], $y$ axis with

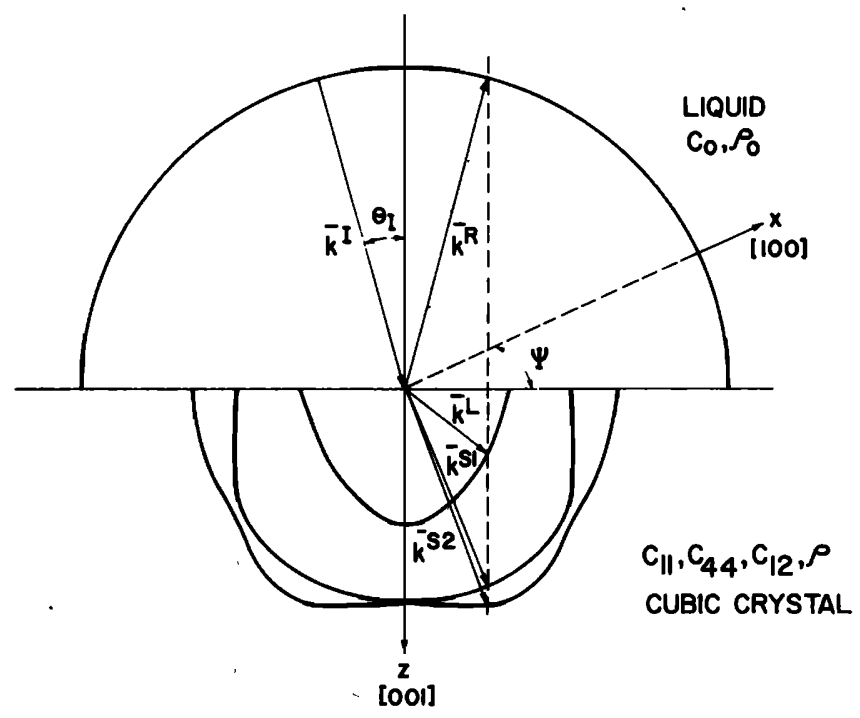

FIG. 1. Acoustic plane-wave scattering at a liquid-cubic-crystal interface. Wave vectors along with slowness surfaces are shown. Interface is the (001) face of the cubic crystal.
[010], and $z$ axis with [001] direction of a cubic solid. Suppose that a unit amplitude plane wave is incident at the liquid-solid interface at $z=0$. The liquid is characterized by its stiffness constant $c_{0}$ and density $\rho_{0}$, and the cubic solid is characterized by three stiffness constants $c_{11}, c_{12}, c_{44}$, and density $\rho$.

The particle velocity field of the incident wave is written as

$$
\mathbf{v}^{I}=\left(\mathbf{k}^{I} / k_{0}\right) \exp \left(j \mathbf{k}^{I} \cdot \mathbf{r}\right), \quad \text { with } \mathbf{r}=x \hat{x}+y \hat{y}+z \hat{z},
$$

where $\mathrm{k}^{I}$ is the wave vector, $k_{0}$ is the wavenumber in the liquid, and $\hat{x}, \hat{y}$, and $\hat{z}$ represent the unit vectors.

Referring to Fig. 1, one reflected wave in the liquid and three refracted -one quasilongitudinal $(\mathrm{QL})$ and two quasishear (QS)-waves in the solid can be present. The corresponding velocity.fields can be written as

$$
\begin{aligned}
& \nabla^{R}=R\left(\mathrm{k}^{R} / k_{0}\right) \exp \left(j \mathbf{k}^{R} \cdot r\right), \\
& \nabla^{L}=L \gamma^{L} \exp \left(j \mathbf{k}^{L} \cdot r\right), \\
& \nabla^{s 1}=S 1 \gamma^{S 1} \exp \left(j \mathbf{k}^{S 1} \cdot r\right), \\
& \nabla^{S 2}=S 2 \gamma^{S 2} \exp \left(j \mathbf{k}^{S 2} \cdot r\right),
\end{aligned}
$$

where $\mathbf{k}^{R}, \mathbf{k}^{L}, \mathbf{k}^{S 1}$, and $\mathbf{k}^{S 2}$ are the wave vectors of the reflected wave in the liquid and of the QL and two QS waves in the solid, respectively. $R, L, S 1$, and $S 2$ stand for the complex amplitudes of the respective waves. $\gamma^{L}, \gamma^{S 1}$, and $\gamma^{S 2}$ are the unity polarization vectors of the refracted waves. In general $\gamma^{L}$ is not in the same direction as $\mathrm{k}^{L}$ for a QL wave, and $\gamma^{S_{1}}$ and $\gamma^{s 2}$ are not necessarily normal to $k^{s 1}$ and $k^{s 2}$ for QS waves.

Using the abbreviated subscript notation of Auld ${ }^{5}$ the boundary conditions on the velocity and stress become

$$
\begin{aligned}
& \left.\left(v_{z}^{I}+v_{z}^{R}\right)\right|_{z=0}=\left.\left(v_{z}^{L}+v_{z}^{S 1}+v_{z}^{S 2}\right)\right|_{z=0}, \\
& \left.\left(T_{J}^{I}+T_{J}^{R}\right)\right|_{z=0}=\left.\left(T_{J}^{L}+T_{J}^{S 1}+T_{J}^{S 2}\right)\right|_{z=0}, \quad J=3,4,5 .
\end{aligned}
$$

First boundary condition gives

$$
\mathbf{k}^{I} \cdot \mathbf{r}=\mathbf{k}^{R} \cdot \mathbf{r}=\mathbf{k}^{L} \cdot \mathbf{r}=\mathbf{k}^{S 1} \cdot \mathbf{r}=\mathbf{k}^{S 2} \cdot \mathbf{r}, \quad \text { at } z=0
$$

or

$$
\begin{aligned}
& k_{x}^{I}=k_{x}^{R}=k_{x}^{L}=k_{x}^{S 1}=k_{x}^{S 2}=l_{x} k_{0}, \\
& k_{y}^{I}=k_{y}^{R}=k_{y}^{L}=k_{y}^{S 1}=k_{y}^{S 2}=l_{y} k_{0},
\end{aligned}
$$

where $l_{x}$ and $l_{y}$ are direction cosines of the incident wave. 
Knowing the $x$ and $y$ components of the wave vector of all the possible waves, we now have to determine the $z$ components. In the liquid we have

$$
\begin{aligned}
& k_{z}^{I}=\left(k_{0}^{2}-k_{x}^{2}-k_{y}^{2}\right)^{1 / 2}=k_{0}\left(1-l_{x}^{2}-l_{y}^{2}\right)^{1 / 2}, \\
& \text { and } k_{z}^{R}=-k_{z}^{I} \text {. }
\end{aligned}
$$

The propagation characteristics of plane waves in the anisotropic solid can be found by the Christoffel equation:

$$
k^{2} \Gamma_{i j} v_{j}=\rho \omega^{2} v_{i},
$$

where $\Gamma_{i j}$ is the Christoffel matrix. ${ }^{5}$ A dispersion relation can be obtained by setting the characteristic determinant equal to zero:

$$
\left|k^{2} \Gamma_{i j}\left(l_{x}, l_{y}, l_{z}\right)-\rho \omega^{2} \delta_{i j}\right|=0 .
$$

From this $k^{2} / \omega^{2}$ can be solved through a third-order equation if the direction cosines of the propagation direction $\left(l_{x}, l_{y}, l_{z}\right)$ are known. Since the direction of the $k$ vector in the solid side is still unknown, we prefer to write Eq. (3) in the following form:

$$
\left|\Gamma_{i j}\left(k_{0} l_{x}, k_{0} l_{y}, k_{z}\right)-\rho \omega^{2} \delta_{i j}\right|=0,
$$

which follows from the property of the Christoffel matrix that $k^{2} \Gamma_{i j}\left(l_{x}, l_{y}, l_{z}\right)=\Gamma_{i j}\left(k_{x}, k_{y}, k_{z}\right)$. In general, Eq. (4) gives a sixth-order equation in $k_{z}{ }^{6}$ The six roots of $k_{z}$ define those wave vectors that can satisfy the condition of having equal components of phase velocity parallel to the boundary plane.

For a cubic crystal Eq. (4) can be written as the square of a cubic polynomial. Hence we have to solve only a thirdorder equation:

$$
\left[\begin{array}{ll}
h_{x}+c_{44}\left(h_{y}+h_{z}\right) / c_{11}-1 & \left(c_{12}+c_{44}\right)\left(h_{x} h_{y}\right)^{1 / 2} / c_{11} \\
\left(c_{12}+c_{44}\right)\left(h_{x} h_{y}\right)^{1 / 2} / c_{11} & h_{y}+c_{44}\left(h_{x}+h_{z}\right) / c_{11}-1 \\
\left(c_{12}+c_{44}\right) h_{x}^{1 / 2} \xi / c_{11} & \left(c_{12}+c_{44}\right) h_{y}^{1 / 2} \xi / c_{11}
\end{array}\right.
$$

From this equation we find the six possible polarizations. Three of these should be selected by taking the Poynting vector into consideration. This vector does not generally coincide with the normal to the wave front in anisotropic media, but it is always perpendicular to the tangent to the slowness surface at the end point of the $k$ vector. For the refracted waves the $z$ component of the Poynting vector is given by

$$
P_{z}=-(K / 2)\left(\gamma_{x}^{*} T_{5}+\gamma_{y}^{*} T_{4}+\gamma_{z}^{*} T_{3}\right),
$$

where $K$ is the square modulus of the wave amplitude (e.g., $\left.|L|^{2}\right)$, and

$$
\begin{aligned}
& T_{3}=-c_{12}\left(\rho / c_{11}\right)^{1 / 2}\left(h_{x}^{1 / 2} \gamma_{x}+h_{y}^{1 / 2} \gamma_{y}\right)-\left(\rho c_{11}\right)^{1 / 2} \xi \gamma_{z}, \\
& T_{4}=-c_{44}\left(\rho / c_{11}\right)^{1 / 2}\left(\xi \gamma_{y}+h_{y}^{1 / 2} \gamma_{z}\right), \\
& T_{5^{5}}=-c_{44}\left(\rho / c_{11}\right)^{1 / 2}\left(\xi \gamma_{x}+h_{x}^{1 / 2} \gamma_{z}\right) .
\end{aligned}
$$

$P_{z}$ must be positive real in order to have a power flow with a positive component in the $z$ direction. It has to be negative imaginary to get an evanescent wave which cannot carry any energy and which vanishes as $z$ goes to infinity. Note that a

$$
\begin{aligned}
& \left(c_{44} / c_{11}\right)^{2} h_{z}^{3}+\left[A\left(h_{x}+h_{y}\right)-C\right] h_{z}^{2} \\
& \quad+\left[A\left(h_{x}^{2}+h_{y}^{2}\right)+B h_{x} h_{y}-D\left(h_{x}+h_{y}\right)+E\right] h_{z} \\
& \quad+\left[\left(c_{44} / c_{11}\right)^{2}\left(h_{x}^{3}+h_{y}^{3}\right)+A\left(h_{x}^{2} h_{y}+h_{x} h_{y}^{2}\right)\right. \\
& \left.\quad-C\left(h_{x}^{2}+h_{y}^{2}\right)-D h_{x} h_{y}+E\left(h_{x}+h_{y}\right)-1\right]=0,
\end{aligned}
$$

where

$$
h_{x}=\frac{\rho_{0} c_{11}}{\rho c_{0}} l_{x}^{2}, \quad h_{y}=\frac{\rho_{0} c_{11}}{\rho c_{0}} l_{y}^{2}, \quad h_{z}=\frac{c_{11} k_{z}^{2}}{\rho \omega^{2}}
$$

and

$$
\begin{aligned}
A= & \left(c_{11} c_{44}^{2}+c_{11}^{2} c_{44}-c_{44} c_{12}^{2}-2 c_{44}^{2} c_{12}\right) / c_{11}^{3}, \\
B= & \left(c_{11}^{3}+4 c_{44}^{3}+2 c_{12}^{3}-3 c_{11} c_{12}^{2}-6 c_{11} c_{12} c_{44}\right. \\
& \left.+6 c_{12}^{2} c_{44}+6 c_{12} c_{44}^{2}\right) / c_{11}^{3}, \\
C= & \left(2 c_{11} c_{44}+c_{44}^{2}\right) / c_{11}^{2}, \\
D= & \left(c_{11}^{2}+2 c_{44}^{2}-c_{12}^{2}+2 c_{11} c_{44}-2 c_{12} c_{44}\right) / c_{11}^{2}, \\
E= & \left(c_{11}+2 c_{44}\right) / c_{11} .
\end{aligned}
$$

Three roots for $h_{z}$ lead to six roots for $k_{z}$ because

$$
k_{z}=\left(\rho / c_{11}\right)^{1 / 2} \omega \xi \text {, where } \xi=\mp h_{z}^{1 / 2} \text {. }
$$

Once the six roots have been determined, the polarizations of the refracted waves $\left(\gamma^{L}, \gamma^{S 1}, \gamma^{S 2}\right)$ must be calculated by finding the eigenvectors of the Christoffel equation. When the eigenvalues are substituted, the Christoffel equation for a cubic medium takes the form

$\left.\begin{array}{l}\left(c_{12}+c_{44}\right) h_{x}^{1 / 2} \xi / c_{11} \\ \left(c_{12}+c_{44}\right) h_{y}^{1 / 2} \xi / c_{11} \\ h_{z}+c_{44}\left(h_{x}+h_{y}\right) / c_{11}-1\end{array}\right] \times\left[\begin{array}{l}v_{x} \\ v_{y} \\ v_{z}\end{array}\right]=0$

solution with a positive imaginary $\boldsymbol{P}_{\mathbf{z}}$ means an exponentially growing wave and it should be eliminated.

Knowing the polarizations and $k$ vectors of the refracted waves, the boundary conditions in Eq. (2) can be used to find the four unknown amplitudes: $R, L, S 1, S 2$.

\section{RESULTS}

A FORTRAN program has been written to perform the numerical calculations summarized in Sec. I.

In Fig. 2 we show the reflection coefficient $R$ at $\mathrm{H}_{2} \mathrm{O}$ GaAs interface as a function of $\sin \theta_{I}=\left(l_{x}^{2}+l_{y}^{2}\right)^{1 / 2}$ for $\psi=28^{\circ}\left[\psi=\tan ^{-1}\left(l_{y} / l_{x}\right)\right]$. In the same figure the "slowness surfaces" are also shown. Note that these slowness curves are plotted in an unusual manner. Horizontal scale is $\sin \theta_{I}$ so that the curves can be compared with the reflection coefficient curves below them easily. The vertical scale is the unitless quantity $\xi$ which was defined before. We can identify the different regions as follows: For $\sin \theta_{I}<0.296$ one quasilongitudinal (QL) and two quasishear (QS) waves can exist in the solid medium. Since there is no evanescent wave 

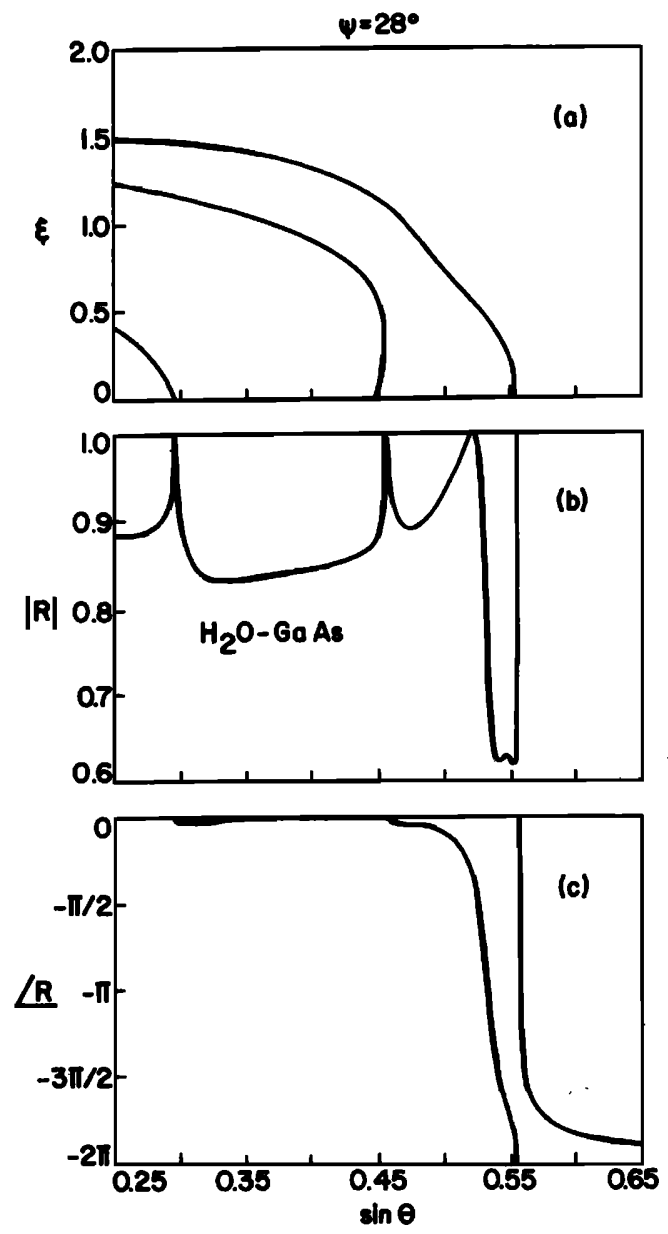

FIG. 2. Slowness surfaces (a) of GaAs for waves propagating in a plane oriented $25^{\circ}$ with respect to a cube face. Reflectance function amplitude (b) and phase (c) at $\mathrm{H}_{2} \mathrm{O}-\mathrm{GaAs}(001)$ interface for the same incidence plane. Elastic parameters of $\mathrm{H}_{2} \mathrm{O}$ and GaAs: $c_{0}=0.2277 \times 10^{10} \mathrm{~N} / \mathrm{m}^{2}$, $\rho_{0}=1 \times 10^{3} \mathrm{~kg} / \mathrm{m}^{3}, \quad c_{11}=11.88 \times 10^{10} \mathrm{~N} / \mathrm{m}^{2}, \quad c_{44}=5.94 \times 10^{10} \mathrm{~N} / \mathrm{m}^{2}$, $c_{12}=5.38 \times 10^{10} \mathrm{~N} / \mathrm{m}^{2}, \rho=5.307 \times 10^{3} \mathrm{~kg} / \mathrm{m}^{3}$. Piezoelectricity neglected.

the phase of the reflection coefficient is zero. For $0.296<\sin \theta_{I}<0.451$ a QL wave cannot propagate, but to satisfy the boundary conditions an evanescent $Q L$ wave must be present. Hence the reflection coefficient phase will be nonzero in this range. This inhomogeneous surface wave may be thought of as a wave that stores the incident energy for a fraction of period equal to the phase change and then reradiates this energy into the reflected waves. ${ }^{6}$ For a very narrow range of incident angles, $0.451<\sin \theta_{I}<0.455$, three QS waves can exist in the solid medium as suggested by the slowness curves. For this case boundary conditions can be satisfied without having to impose an evanescent wave; therefore the reflection coefficient phase is zero in this narrow range. For $0.455<\sin \theta_{I}<0.554$, only one QS wave can propagate. Boundary conditions require one $\mathrm{QL}$ and one $\mathrm{QS}$ evanescent wave. Hence a Rayleigh surface wave may be excited in this range. The reflection coefficient phase undergoes a large shift centered at $\sin \theta_{I}=0.532$. This transition in the phase function corresponds to a Rayleigh wave excitation. This wave is known as a "pseudo-surface wave."7 For $\sin \theta_{I}>0.554$, no wave can propagate. Hence the last QS
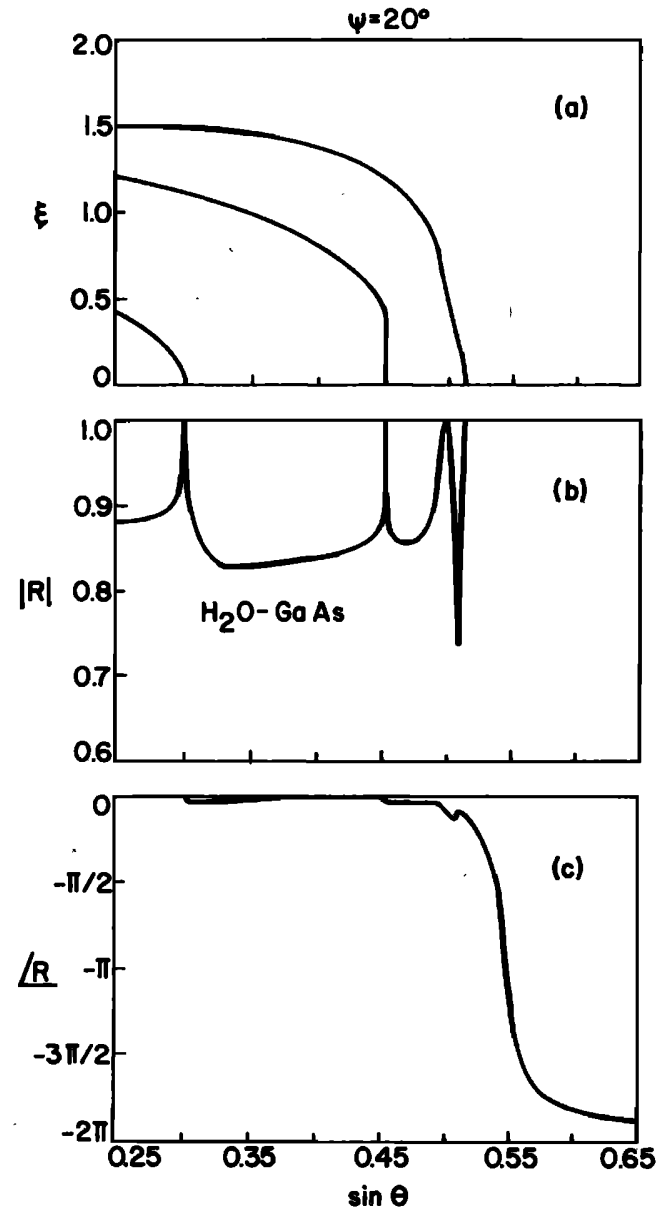

FIG. 3. Slowness surfaces (a) of GaAs for waves propagating in a plane oriented $20^{\circ}$ with respect to a cube face. Reflectance function amplitude (b) and phase (c) at $\mathrm{H}_{2} \mathrm{O}-\mathrm{GaAs}(001)$ interface for the same incidence plane.

wave becomes evanescent as well. Here there is another possibility that Rayleigh condition could be satisfied. The second transition indicates that there is indeed a Rayleigh wave excitation at $\sin \theta_{I}=0.5545$, and this wave is sometimes called a "generalized Rayleigh wave." For this wave the particle motion is not in a plane normal to the surface. A surprising fact to note is that the reflection coefficient is about $\mathbf{0 . 6 2}$ for $0.536<\sin \theta_{1}<0.552$.

The same set of curves is shown for $\psi=20^{\circ}$ in Fig. 3. For this case, there is only one transition in the phase curve. Therefore the pseudo surface wave does not exist for this orientation. Actually it has been shown that ${ }^{7}$ for the propagation on the (001) plane of a cubic medium, the pseudosurface wave is present only for a range of directions near [110] axis.

To investigate the possibility of a null in the reflection coefficient several other orientation angles $(\psi)$ have been tried. The results are shown in Fig. 4. For $\psi=25^{\circ}$ the reflection coefficient magnitude at $\sin \theta_{I}=0.53$ is almost zero! The null in the reflection coefficient is not unique to $\mathrm{H}_{2} \mathrm{O}$ GaAs interface. Several other cubic crystals have been investigated and they all have nulls albeit at different angles. Figure 5 depicts the reflection coefficient for $\mathrm{H}_{2} \mathrm{O}$-InAs interface where the null is displayed for $\psi=24^{\circ}$ and $\sin \theta_{I}$ 


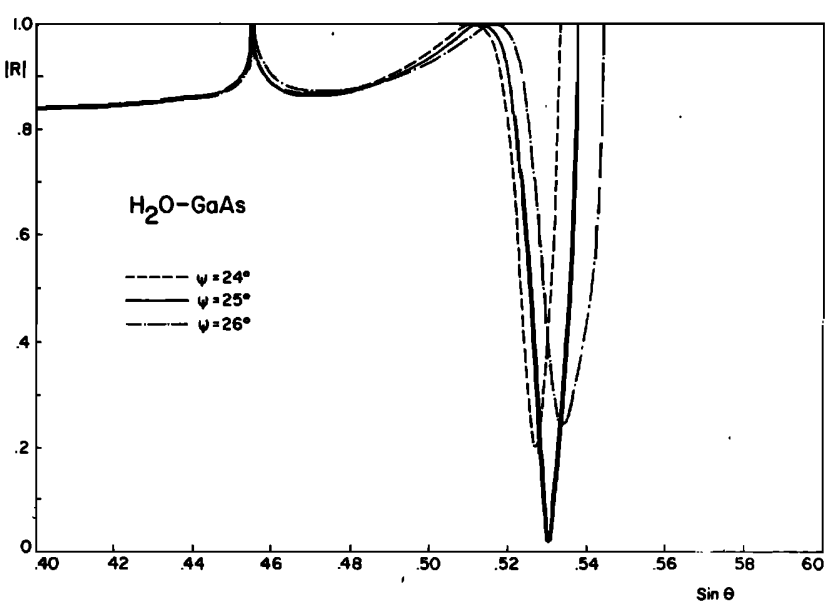

FIG. 4. Reflectance function amplitude for various orientations at $\mathrm{H}_{2} \mathrm{O}$ GaAs interface.

$=0.68$. Figure 6 shows the results for $\mathrm{H}_{2} \mathrm{O}-\mathrm{Cu}$ interface. In this case a very narrow null is obtained around $\psi=25.1^{\circ}$ and $\sin \theta=0.665$.

The perfect null angle can be found by changing $\psi$ and $\sin \theta_{I}$ appropriately using perhaps an iterative procedure. The null angle is in the region where only one QS wave can propagate in the cubic medium. Therefore at this angle there is a perfect power transfer from the incident wave in the liquid to a QS wave in the solid. We note that this impedance match occurs for a very narrow range of angle (in both $\theta_{I}$ and $\psi$ directions).

To verify the validity of the program conservation of energy is checked at each angle. Incident power is calculated using normal component of the Poynting vector. This is compared with the sum of normal real components of Poynting vectors for reflected and refracted waves. In the region of interest where only one QS wave can exist in the solid only the Poynting vector for this QS wave is real. Evanescent waves give rise to complex valued Poynting vectors.

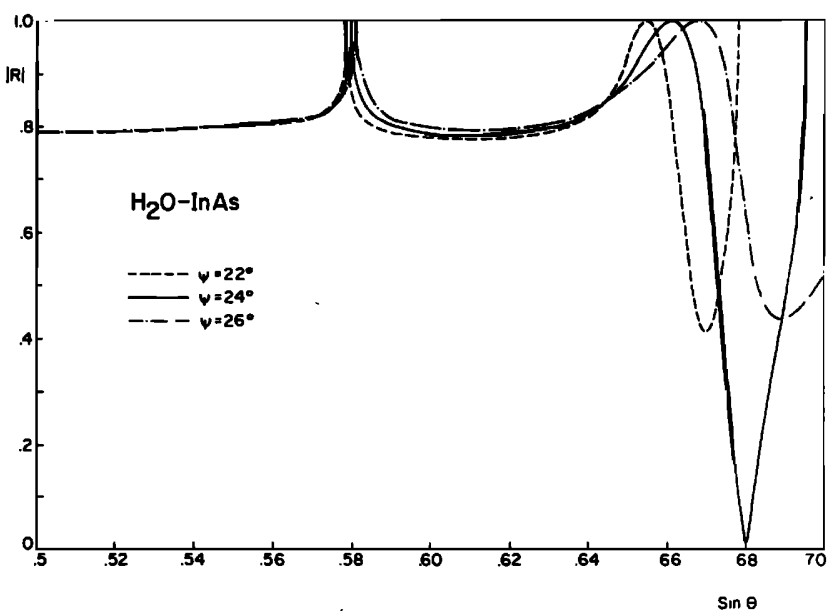

FIG. 5. Reflectance function amplitude for various orientations at $\mathrm{H}_{2} \mathrm{O}$ InAs interface. Elastic parameters of InAs: $c_{11}=8.329 \times 10^{10} \mathrm{~N} / \mathrm{m}^{2}$, $c_{44}=3.959 \times 10^{10} \mathrm{~N} / \mathrm{m}^{2}, c_{12}=4.526 \times 10^{10} \mathrm{~N} / \mathrm{m}^{2}, \rho=5.672 \times 10^{3} \mathrm{~kg} / \mathrm{m}^{3}$.

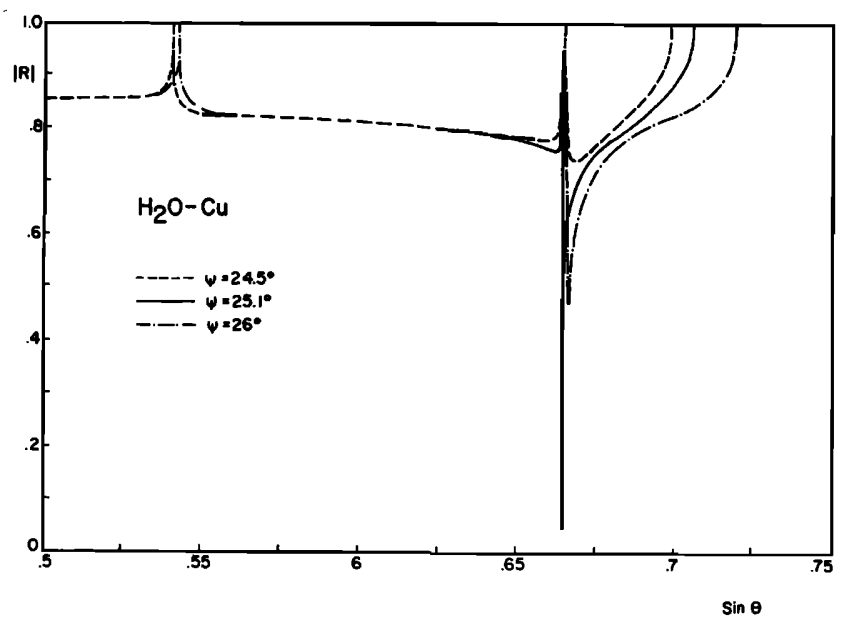

FIG. 6. Reflectance function amplitude for various orientations at $\mathrm{H}_{2} \mathrm{O}-\mathrm{Cu}$ interface. Elastic parameters of $\mathrm{Cu}$ : $c_{11}==17.02 \times 10^{10} \mathrm{~N} / \mathrm{m}^{2}$, $c_{44}=7.51 \times 10^{10} \mathrm{~N} / \mathrm{m}^{2}, c_{12}=12.3 \times 10^{10} \mathrm{~N} / \mathrm{m}^{2}, \rho=8.936 \times 10^{3} \mathrm{~kg} / \mathrm{m}^{3}$.

\section{DISCUSSION}

To understand the reason why one may have an impedance match between a low-impedance liquid and a seemingly high-impedance anisotropic solid we shall look at a degenerate case, namely an isotropic solid. This case is so simple that analytic expressions are available. The reflection coefficient can be written as ${ }^{4}$

$$
R=\left(Z_{L}+Z_{S}-Z_{0}\right) /\left(Z_{L}+Z_{S}+Z_{0}\right),
$$

where

$$
\begin{aligned}
& Z_{0}=\left(c_{0} \rho_{0}\right)^{1 / 2} / \cos \theta_{I}, \\
& Z_{L}=\left[\left(c_{11} \rho\right)^{1 / 2} / \cos \theta_{L}\right] \cos ^{2} 2 \theta_{s}, \\
& Z_{s}=\left[\left(c_{44} \rho\right)^{1 / 2} / \cos \theta_{S}\right] \sin ^{2} 2 \theta_{s},
\end{aligned}
$$

with $\theta_{L}$ and $\theta_{s}$ being the refraction angles as determined by the Snell's law

$$
\frac{\sin \theta_{I}}{\left(c_{0} / \rho_{0}\right)^{1 / 2}}=\frac{\sin \theta_{L}}{\left(c_{11} / \rho\right)^{1 / 2}}=\frac{\sin \theta_{s}}{\left(c_{44} / \rho\right)^{1 / 2}} .
$$

Making the analogy between the reflection process in acoustics with the reflection process in electrical transmission lines one has

$R=\left(Z_{\text {load }}-Z_{0}\right) /\left(Z_{\text {load }}+Z_{0}\right)$, where $Z_{\text {load }}=Z_{L}+Z_{S}$.

$Z_{L}$ is the impedance contribution of longitudinal waves and $Z_{S}$ is that of the shear waves. Contributions become imaginary when the corresponding wave becomes evanescent. $Z_{\text {load }}$ and $Z_{0}$ for $\mathrm{H}_{2} \mathrm{O}-\mathrm{YIG}$ interface is plotted in Fig. 7. YIG is selected as the solid since it is very nearly isotropic.

$Z_{L}$ has a nonzero value at $\sin \theta_{I}=0$ and approaches to infinity at the longitudinal critical angle (LCA). Beyond this angle $Z_{L}$ has a negative imaginary part. It vanishes when $\sin \theta_{s}=1 / \sqrt{2}$. On the other hand $Z_{s}$ is zero at $\sin \theta_{I}=0$. It goes through a maximum and then it becomes zero at the shear critical angle (SCA). For angles exceeding SCA, $Z_{s}$ is a monotonically increasing positive imaginary number. At an angle slightly above SCA, namely at the Rayleigh critical angle (RCA), $Z_{L}$ and $Z_{s}$ completely cancel each other while one is positive imaginary and the other is negative imagi- 


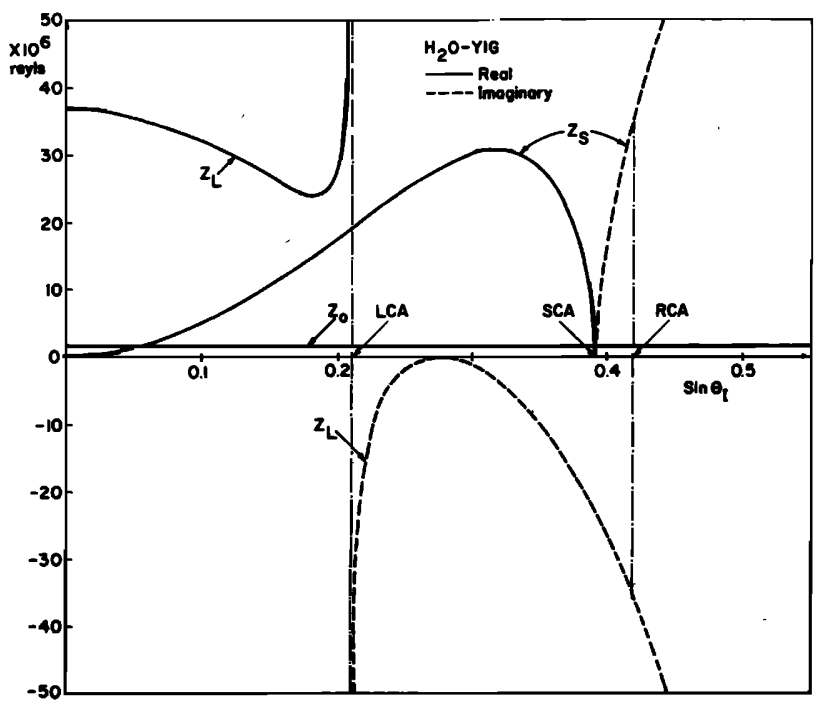

FIG. 7. $Z_{\text {load }}$ and $Z_{0}$ as a function of incidence angle for $\mathrm{H}_{2} \mathrm{O}-\mathrm{YIG}$ interface. Elastic parameters of YIG: $c_{11}=26.8 \times 10^{10} \mathrm{~N} / \mathrm{m}^{2}$, $c_{44}=7.66 \times 10^{10} \mathrm{~N} / \mathrm{m}^{2}, \rho=5.17 \times 10^{3} \mathrm{~kg} / \mathrm{m}^{3}$.

nary. At this point $Z_{\text {load }}=0$ and it is the angle where a surface wave is excited. One should recall that for perfect match $Z_{\text {load }}=Z_{0}$ must be satisfied. Since $Z_{0}$ is always positive real, a match can occur only in the region below LCA. But for most liquid-solid interfaces, this is not possible while the impedances of solids are usually much higher than that of liquids. (There are exceptions when liquid is a heavy metal like mercury or gallium.)

Using the results of isotropic case we propose to write the reflection coefficient for a liquid-anisotropic solid interface as

$$
\begin{aligned}
& R=\left(Z_{\text {load }}-Z_{0}\right) /\left(Z_{\text {load }}+Z_{0}\right), \\
& \text { and } Z_{\text {load }}=Z_{L}+Z_{S 1}+Z_{S 2},
\end{aligned}
$$

where $Z_{L}, Z_{S 1}$, and $Z_{S 2}$ are the impedance contributions of QL and two QS waves, respectively. Although we do not have analytic expressions for these impedances we expect that they behave similarly to those of the isotropic case. But overall behavior should be different since there are three critical angles as opposed to two. Each contribution will become imaginary when the corresponding critical angle is exceeded.

There are four distinct regions separated by the three critical angles. In region $I$ [below the quasilongitudinal critical angle $(\mathrm{QLCA})] Z_{L}, Z_{S 1}$, and $Z_{S 2}$ are all positive real. In region II where QLCA is exceeded $Z_{L}$ becomes negative imaginary while $Z_{S 1}$ and $Z_{S 2}$ are still positive real. In region III, the first QS critical angle (QSCA1) is exceeded and $Z_{S_{1}}$ becomes positive imaginary. In this region (between QSCA1 and QSCA2) $Z_{S 1}$ and $Z_{L}$ are imaginary with opposite signs while $Z_{S 2}$ is positive real. One may expect a cancellation of $Z_{S 1}$ and $Z_{L}$ at some angle similar to that which occurred in the isotropic case under Rayleigh critical angle excitation. If this angle exists it will be the pseudo-surface wave angle (PSWA). At this angle $Z_{\text {load }}$ is equal to $Z_{S 2}$ and it is positive real. In region IV where all critical angles are exceeded all three contributions to $Z_{\text {load }}$ are imaginary. For some cases there may be a fifth region within region II. In this range three $Q S$ waves are excited; therefore $Z_{\text {load }}$ is real again even though QLCA is exceeded. The statements in this paragraph are summarized in Table $I$.

For an impedance match to occur $Z_{\text {load }}$ must be equal to $Z_{0}$. Recall that $Z_{0}$ is always positive real. Therefore a match may occur only if $Z_{\text {load }}$ is positive real. This is satisfied in region $I$, in region III at PSWA, and in region V if it exists. For most liquid-solid interfaces a match cannot occur in region I since $Z_{L}$ is much higher than $Z_{0}$. Similarly region $\mathrm{V}$ is an unlikely region for a match for $Z_{\text {load }}$ has three positive real (possibly large) components in this region. On the other

\begin{tabular}{|c|c|c|c|c|c|c|c|}
\hline Incidence angle & Region & $Z_{L}$ & $Z_{s !}$ & $Z_{s 2}$ & $Z_{\text {load }}$ & $\begin{array}{l}Z_{\text {load }}=Z_{0} \\
\text { possible? }\end{array}$ & $\begin{array}{l}Z_{\text {load }}=Z_{0} \\
\text { likely? }\end{array}$ \\
\hline$\theta_{I}=0$ & I & $\begin{array}{l}\text { positive } \\
\text { real }\end{array}$ & 0 & 0 & $\begin{array}{l}\text { positive } \\
\text { real }\end{array}$ & yes & no \\
\hline$\theta_{\boldsymbol{l}}<\mathrm{QLCA}$ & I & $\begin{array}{l}\text { positive } \\
\text { real }\end{array}$ & $\begin{array}{l}\text { positive } \\
\text { real }\end{array}$ & $\begin{array}{l}\text { positive } \\
\text { real }\end{array}$ & $\begin{array}{l}\text { positive } \\
\text { real }\end{array}$ & yes & no \\
\hline QLCA $<\theta_{I}<$ QSCA1 & II & $\begin{array}{l}\text { negative } \\
\text { imag }\end{array}$ & $\begin{array}{l}\text { positive } \\
\text { real }\end{array}$ & $\begin{array}{l}\text { positive } \\
\text { real }\end{array}$ & complex & $\begin{array}{l}\text { no } \\
\text { (unless } \\
Z_{L}=0 \text { ) }\end{array}$ & no \\
\hline QSCA1 $-\epsilon<\theta_{I}<$ QSCA1 & $\begin{array}{l}V \\
\text { if exists }\end{array}$ & $\begin{array}{l}\text { positive } \\
\text { real }\end{array}$ & $\begin{array}{l}\text { positive } \\
\text { real }\end{array}$ & $\begin{array}{l}\text { positive } \\
\text { real }\end{array}$ & $\begin{array}{l}\text { positive } \\
\text { real }\end{array}$ & yes & no \\
\hline QSCA1 $<\theta_{l}<$ QSCA2 & III & $\begin{array}{l}\text { negative } \\
\text { imag }\end{array}$ & $\begin{array}{l}\text { positive } \\
\text { imag }\end{array}$ & $\begin{array}{l}\text { positive } \\
\text { real }\end{array}$ & complex & no & no \\
\hline$\theta_{I}=$ PSWA & III & $\begin{array}{l}\text { negative } \\
\text { imag }\end{array}$ & $\begin{array}{l}\text { positive } \\
\text { imagl }=\end{array}$ & $\begin{array}{l}\text { positive } \\
\text { real }\end{array}$ & $\begin{array}{l}\text { positive } \\
\text { real }\end{array}$ & yes & yes \\
\hline$\theta_{I}>$ QSCA2 & IV & $\begin{array}{l}\text { negative } \\
\text { imag }\end{array}$ & $\begin{array}{l}\text { positive } \\
\text { imag }\end{array}$ & $\begin{array}{l}\text { positive } \\
\text { imag }\end{array}$ & imaginary & no & no \\
\hline
\end{tabular}

TABLE I. Expected behavior of $Z_{L}, Z_{S 1}$, and $Z_{S 2}$ as a function of incidence angle. 
hand at PSWA in region III a match may be possible. If $Z_{S 2}=Z_{0}$ is satisfied, all the incident power will be coupled to a QS wave inside the solid. Unfortunately we do not have an analytic expression for $\boldsymbol{Z}_{\boldsymbol{S} 2}$. Its value should depend on $\sin \theta_{I}$ as well as the orientation angle $\psi$. We assume that it behaves like $Z_{s}$ of isotropic case (as shown in Fig. 7). With this assumption one may argue that $Z_{s 2}=Z_{0}$ will be satisfied for some $\theta_{I}=\theta_{c}$ at any given orientation angle $\psi$, because the corresponding curves in isotropic case intersect each other (see Fig. 7). A match condition will be achieved if $\theta_{c}$ coincides with PSWA. This coincidence-if it occurswill take place at a particular orientation $\psi_{c}$. Therefore a null in the reflection coefficient will be seen if $Z_{S 2}=Z_{0}$ is satisfied at a pseudo-surface wave angle. ${ }^{8}$ On the other hand if $Z_{s 2}=Z_{0}$ is satisfied at an orientation where there exists no pseudo-surface waves, a null will not be present.

The heuristic argument above does not guarantee that there is an angle of perfect match for every liquid-cubicsolid interface. It merely shows that if a pseudo-surface wave exists, a perfect coupling from the incident longitudinal wave in the liquid to a QS wave in the solid might occur for some orientation $\psi_{c}$.

The conclusions reached above could be generalized to other faces of cubic crystals and to any face of any anisotropic solid for we have not used cubic symmetry in the arguments. Therefore one may expect to find reflection coefficient nulls for any anisotropic medium as long as pseudo-surface waves exist on the surface of interest.

\section{CONCLUSION}

The results of numerical calculations for the reflection coefficient at a liquid-cubic-solid interface are presented. The calculations are performed only for the (001) face of the solid. A null in the reflection coefficient is detected for a particular orientation. At this angle all the incident power in the liquid is coupled into a quasishear wave in the solid. Various computer runs showed that this is not unique to certain materials. An explanation is presented for the presence of null in the reflection coefficient using an impedance argument. This argument shows that if there is a null in the reflection coefficient it will be at an angle associated with the pseudo-surface wave. The same argument concludes that this null is not unique to the (001) face of cubic materials; other materials with noncubic symmetry might have nulls in the reflection coefficient as well.

'F. L. Becker and R. L. Richardson, J. Acoust. Soc. Am. 51, 1609 (1972). ${ }^{2}$ M. A. Breazeale, L. Adler, and G. W. Scott, J. Appl. Phys. 48, 530 (1977). ${ }^{3}$ T. J. Plona, M. Behravesh, and W. G. Mayer, J. Acoust. Soc. Am. 56, 1773 (1974).

${ }^{4}$ L. M. Brekhovskikh, Waves in Layered Media (Academic, New York, 1960).

${ }^{5}$ B. A. Auld, Acoustic Fields and Waves in Solids, Vols. 1 and 2 (WileyInterscience, New York, 1973).

${ }^{6}$ E. G. Henneke, II, J. Acoust. Soc. Am. 51, 210 (1972).

${ }^{7}$ T. C. Lim and G. W. Farnell, J. Acoust. Soc. Am. 45, 845 (1969).

${ }^{8}$ F. R. Rollins, T. C. Lim, and G. W. Farnell, Appl. Phys. Lett. 12, 236 (1968) calculated the pseudo-surface velocities for $(100)$ face of copper. The velocity at $\psi=25.1^{\circ}$ agrees very well with the null angle shown in Fig. 6 . 